\title{
Electrically Evoked Auditory Brainstem Response by Using Bionic Auditory Membrane in Guinea Pigs*
}

\author{
Hirofumi SHINTAKU**, Takatoshi INAOKA***, Takayuki NAKAGAWA***, \\ Satoyuki KAWANO** and Juichi ITO*** \\ ** Department of Mechanical Science and Bioengineering, \\ Graduate School of Engineering Science, Osaka University \\ 1-3 Machikaneyama, Toyonaka, Osaka 560-8531, Japan \\ E-mail: kawano@me.es.osaka-u.ac.jp \\ *** Department of Otolaryngology, Head and Neck Surgery, \\ Graduate School of Medicine, Kyoto University \\ 54 Kawara, Shogoin, Sakyo, Kyoto 606-8507, Japan
}

\begin{abstract}
Here, we report the measurement of an eABR (electrically evoked auditory brainstem response) by using a custom-developed BAM (bionic auditory membrane) for a novel artificial cochlear system. The BAM is an acoustic sensor designed as a trapezoidal and flexible membrane made of a piezoelectric material to convert acoustic waves to electrical signals with frequency selectivity. The signal from the BAM was used as an electrical source to stimulate the auditory nerves in a cochlea. The stimulating characteristics were investigated by measuring the eABR in guinea pigs. The results showed that the developed system could realize the perception of peak sound pressure levels and frequency of the acoustic wave. Consequently, we accumulated fundamental knowledge for developing a fully implantable artificial cochlea based on the BAM.
\end{abstract}

Key words: Cochlear Implants, Acoustic MEMS Sensor, Piezoelectric Device, Animal Test, Auditory Brainstem Response

\section{Introduction}

Cochlear implants are medical devices that have recently been used to treat sensorineural hearing loss. Current and conventional systems consist of an implant as well as an extracorporeal device [1,2] in which acoustic waves are detected by a microphone and analyzed based on frequency by a digital processor. The analyzed information is transferred to the implant as electrical signals, and electrical stimulation is provided to the auditory nerves through multi-channel electrodes inserted in the cochlea of the inner ear. By taking advantage of the intrinsic tonotopic relationship between the frequency and the location of auditory nerves, the frequency of the acoustic wave is decoded as the site of cochlear stimulation to yield the appropriate frequency information for auditory nerve stimulation. Although conventional cochlear implants can realize the fundamental perception of sounds, they still have disadvantages in terms of their relatively high energy consumption, limited tone levels because of the small number of electrodes, and indispensability of extracorporeal devices.

Recent progress in microfabrication technology has expanded the application

of biomedical devices [3-5]. Several microdevices that mimic the mechanical cochlear system have been proposed to implement a cochlea-like frequency analyzer [6-12]. In 
these devices, the frequency analyses are achieved by a membrane [7, 12], beam array $[8,11]$, and membrane with beams $[6,9,10]$, which flexibly vibrate by applying sound. In a previous study, we proposed a BAM (bionic auditory membrane) as the sensor for a novel artificial cochlear system [7]. The BAM-a flexible and trapezoidal membrane made of a piezoelectric material known as PVDF (polyvinylidenedifluoride) - is designed to mimic the frequency selectivity mechanism of the basilar membrane in a biological cochlea and realize acoustic-electric conversion without supplying energy. Discrete and thin-film electrodes are fabricated on the surface by UV-lithography and etching technologies. Thus, the BAM can convert acoustic waves to electrical signals by using the piezoelectric effect. In addition, the long and trapezoidal geometry of the BAM contributes to the frequency analysis by changing the local characteristic frequency (CF) of vibration along the longitudinal direction. That is, by applying an acoustic wave, a local area of the BAM where the CF matches the frequency of the acoustic wave predominantly vibrates, resulting in relatively large vibration amplitudes and large mechanical deformations in this area. Consequently, the frequency is decoded as the electrode site that gives large electrical signals.

Very recently, we reported preliminary animal tests in guinea pigs and discussed the fundamental principles of using the BAM as the sensor for a novel artificial cochlea [13]. In this study, we report a system consisting of the BAM and an electrical circuit for systematic animal tests based on the eABR (electrically evoked auditory brainstem response) [14] and present a detailed discussion on the decoding of sound pressure level and frequency. In the system, we use the electrical signals obtained from the BAM as sources for electrical stimuli to the auditory nerves in a cochlea. The stimulating characteristics are then investigated by the eABR. Consequently, brainstem responses are successfully observed by using the developed system. Furthermore, we find that the BAM can decode sound pressure levels and frequencies of acoustic waves by changing the magnitude of the electrical stimulus.

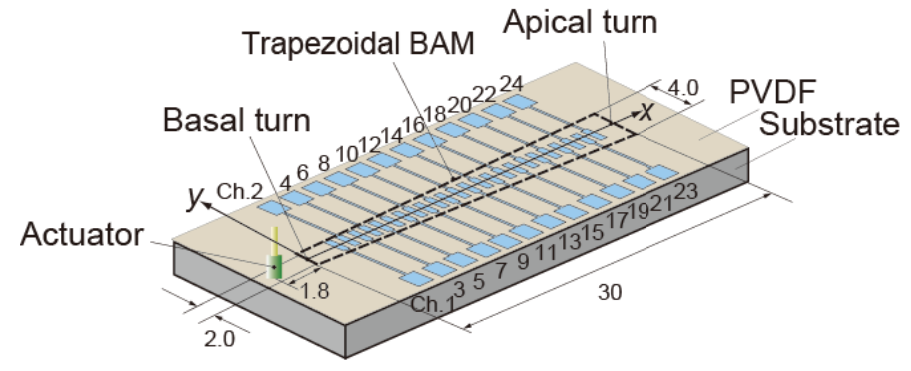

Fig. 1 Schematic of BAM (bionic auditory membrane) for an artificial cochlea (Unit: mm).
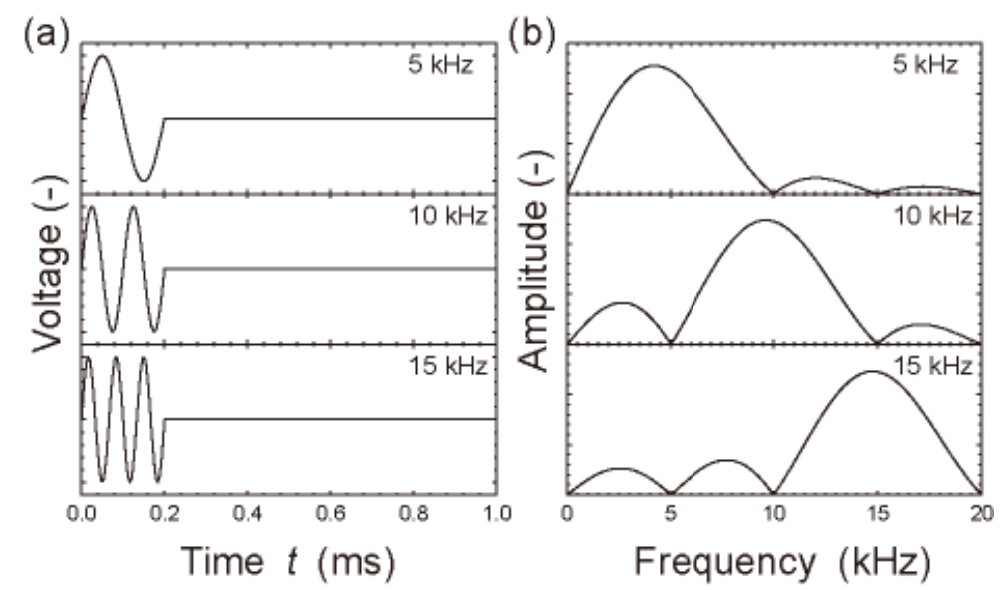

Fig. 2 (a) Applied voltage for generation of pulsed sine waves at base frequencies of 5 , 10, and $15 \mathrm{kHz}$. (b) Fourier spectra for pulsed sine waves. 


\section{Animal Experiments}

\subsection{Generation of Electric Signals by Bionic Auditory Membrane}

Figure 1 shows a schematic of the BAM and coordinate system. The BAM is $40-\mu \mathrm{m}$ thick and $30-\mathrm{mm}$ long with a width range of $2.0-4.0 \mathrm{~mm}$ along the longitudinal direction. The $\mathrm{CF}$, which is determined by the local geometry and the mechanical properties of the BAM, decreases from 19.8 to $6.6 \mathrm{kHz}$ along $x$ in air [7]. Thin-film electrodes are prepared to obtain electrical signals at each CF. The number of the electrodes is designed to be 24 , with the electrodes named Ch. $1-$ Ch. 24 along $x$. The position of Ch. 1 is defined as $x=2.0 \mathrm{~mm}$ which is from the basal turn, and subsequent electrodes are equally spaced with a center-to-center spacing of $\Delta x=1.1 \mathrm{~mm}$.

The basic performance characteristics of the BAM are analyzed through measurement of the electrical signals by using the pulse-echo method. A piezoelectric actuator located at $(x, y)=(-1.8 \mathrm{~mm}, 0 \mathrm{~mm})$ is used to apply pulsed acoustic waves to the BAM. This method allows us to simulate wave propagation from the basal turn to the apical turn on the basilar membrane in a biological cochlea. The magnitude of the acoustic wave is controlled by changing the amplitude of the applied voltage to the actuator; it is described by the pSPL (peak sound pressure level). The value of pSPL, which is for the transient sound pressure, represents the magnitude of the pure tone sound with the same peak height of sound pressure. The relation between the pSPL and the applied voltage to the actuator is correlated by a calibration curve that is obtained in our preliminary experiment under the known pSPL. Figure 2 (a) shows a conceptual waveform of applied voltage to induce sinusoidal acoustic waves lasting for $0.2 \mathrm{~ms}$. The base frequencies of the waves are 5,10 , and $15 \mathrm{kHz}$. That is, the cycles per acoustic wave are 1, 2, and 3, respectively. The corresponding Fourier spectra are shown in Fig. 2 (b). The spectra are mainly distributed in the range of $\pm 5 \mathrm{kHz}$ around each base frequency. These pulsed sine waves enable us to effectively investigate the frequency selectivity of the BAM, taking advantage of the characteristic spectra in Fig. 2 (b). Measurement of the electrical signal from the BAM begins when an acoustic wave is applied to the BAM at $t=0 \mathrm{~ms}$.

(a)
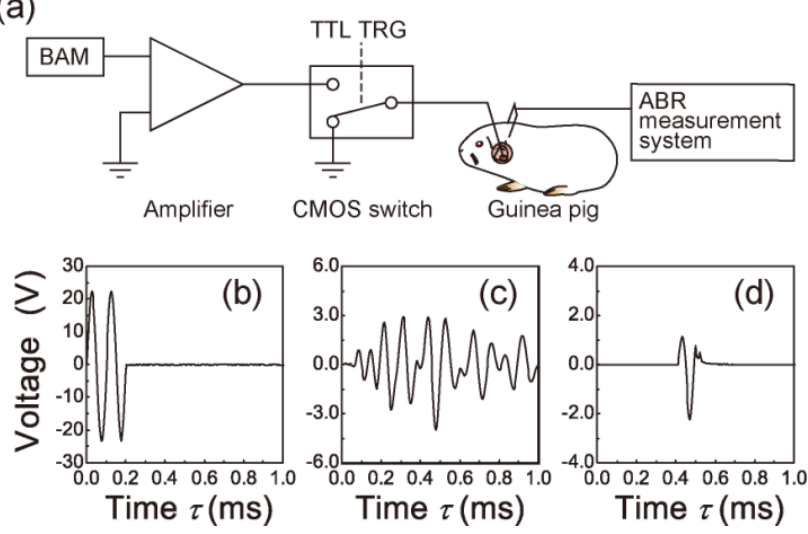

Fig. 3 (a) Schematic of experimental setup for eABR measurement by using BAM. (b) Applied voltage to BAM at base frequency of $10 \mathrm{kHz}$, (c) electrical signal generated by BAM, and (d) electrical stimulus for eABR.

\subsection{Measurement of Electrically Evoked Auditory Brainstem Response by Using Bionic Auditory Membrane}

All experimental procedures followed the National Institutes of Health Guidelines for the Care and Use of Laboratory Animals and were approved by the Animal Experiments Committee at Osaka University and the Institutional Animal Committee at Kyoto University. Animal care was conducted under the supervision of the Institute of Laboratory Animals, 
Graduate School of Medicine, Kyoto University, Japan. In all procedures, the animals were anesthetized with an intramuscular injection of midazolam $(10 \mathrm{mg} / \mathrm{kg})$ and xylazine $(0.01$ $\mathrm{mg} / \mathrm{kg}$ ). Figure 3 shows the electrical circuit and signal processing of a prototype artificial cochlea based on the BAM for eABR measurements. The circuit consists of an amplifier and a CMOS switch, as shown in Fig. 3 (a). The electrical signals from the BAM are used as a source for the electrical stimuli. To effectively stimulate the nerve cells, the electrical signal is amplified $O(100)$-fold. Although the voltage is applied only for $0.2 \mathrm{~ms}$ to drive the actuator (Fig. 3 (b)), the BAM generates an electrical echo signal longer than $0.2 \mathrm{~ms}$ (Fig. 3 (c)). The echo signal is due to reverberation. However, the electrical stimulus should be shorter than $0.5 \mathrm{~ms}$ for superior $\mathrm{S} / \mathrm{N}$ (signal-to-noise) ratios in eABR measurements. Therefore, the CMOS switch is installed between the amplifier and the guinea pig to modify the electrical stimuli in a manner suitable for measurement. The switch is normally off and is turned on for the predetermined duration to extract a biphasic stimulus (Fig. 3 (d)) from the original signal (Fig. 3 (c)). The duration of the on state is controlled by the TTL (transistor-transistor logic) trigger applied to the switch.

The guinea pigs for the experiment were deafened in advance by irreversibly damaging the hair cells with an intramuscular application of kanamycin $(800 \mathrm{mg} / \mathrm{kg})$ followed $2 \mathrm{~h}$ later by an intravenous injection of ethacrynic acid ( $80 \mathrm{mg} / \mathrm{kg}$ ). Deafness was confirmed by standard measurements of the auditory brainstem response (ABR) to a sound. A pair of electrodes made of platinum indium wires was used to electrically stimulate the auditory nerves. One of the electrodes was inserted into the scala tympani of the cochlea near the basal turn, and the other was placed on the bony wall of the cochlea near the basal turn. Dental cement was used to close a bone defect created for electrode insertion. The impedance between two electrodes was measured to be $O(10)$ $\mathrm{k} \Omega$, which varies in different guinea pigs. Brainstem responses to the electrical stimulus were measured by using electrodes attached to the head of the guinea pig. As described above, a single-channel electrode was used for eABR measurement, instead of the multi-channel electrode necessary for realizing frequency selectivity in conventional cochlear implants. One of the 24 channels of the BAM was chosen to be connected to the single-channel electrode for stimulation. The effect of frequency selectivity of the BAM is evaluated by variations in the magnitude of the responses when connecting different channels.

\section{Results and Discussion}

\subsection{Electrical Signals from Bionic Auditory Membrane}

Figure 4 (a) shows the electrical signals obtained from Ch. 2, 10, 18, 20, 22, and 24 by applying a pulsed acoustic wave with a base frequency of $5 \mathrm{kHz}$. The acoustic wave is applied to the BAM for $0.2 \mathrm{~ms}$ from $t=0 \mathrm{~ms}$ by using the actuator. The rise in the signal is delayed by $\sim 0.03 \mathrm{~ms}$ owing to wave propagation from the actuation site to the BAM. Each electrode gives different electrical signals, which can roughly be classified into two trends: those for Ch. 2-18 and those for Ch. 20-24. In Ch. 2-18, the electrical signals rise for 0.2 $\mathrm{ms}$ and decay immediately. This indicates that the local sites for Ch. 2-18 vibrate only when they are forced to do so. In contrast, in Ch. 20-24, the electrical signals continue to be generated for a while longer than $0.2 \mathrm{~ms}$. This indicates that the local sites of Ch. 20-24 continue vibrating owing to reverberation. This difference in trend is due to the relation between the local CF and the acoustic wave spectra. To clarify the detailed mechanism, the electrical signals were subjected to Fourier analysis. The spectra corresponding to the electrical signals in Fig. 4 (a) are obtained as Fig. 4 (b). The spectra for Ch. 20-24 have clear peaks at each frequency, whereas those for Ch. 2-18 do not. Reasonable results are successfully obtained because the observed frequency decreases from 7.11 to $6.21 \mathrm{kHz}$ as the channel number increases from 20 to 24. Compared with our previous results [7], the 
frequency is found to correspond to the local CF of the BAM. The absence of a clear peak in Ch. 2-18 of Fig. 4 (b) can be explained by considering the original spectra of the applied acoustic wave. The magnitude of the applied acoustic wave at a base frequency of $5 \mathrm{kHz}$ is relatively large over the frequency range of $2-8 \mathrm{kHz}$, as shown in Fig. 2 (b). Thus, $\mathrm{Ch}$. $20-24$, whose $\mathrm{CFs}$ are within the range described above, generate relatively large signals. In contrast, the applied acoustic wave has small magnitudes outside of the abovementioned frequency range. Thus, Ch. $2-18$, which have a higher CF than $8 \mathrm{kHz}$, generate small and short signals for the pulsed sine wave at $5 \mathrm{kHz}$.

The electrical signals measured by applying acoustic waves with a base frequency of $15 \mathrm{kHz}$ are shown in Fig. 5 (a). Note that the wave in the period indicated as the actuation has about 2.5 cycles though the applied acoustic wave is 3 cycles. This is due to the delay of the wave to propagate from the actuation point to the measurement point. In contrast to Fig. 4 (a), continuous electrical signals are generated from Ch. $2-10$. This is because the applied acoustic wave includes relatively large-magnitude signals in the range of $12-18 \mathrm{kHz}$ (Fig. 2 (b)) and the $\mathrm{CF}$ values of Ch. 2-10 are in the abovementioned frequency range. Figure 5 (b) shows the Fourier spectra obtained from the signals in Fig. 5 (a). As can be expected, clear peaks are observed in Ch. 2-10. However, the spectra for $\mathrm{Ch}$. 18-24 also show non-trivial peaks. This indicates a disadvantage of the passive BAM in frequency selectivity, which may be improved by introducing an active feedback that mimics the function of outer hair cells. From these results, the basic characteristics of acoustic-electric conversion and frequency selectivity are clarified by applying pulsed acoustic waves. 

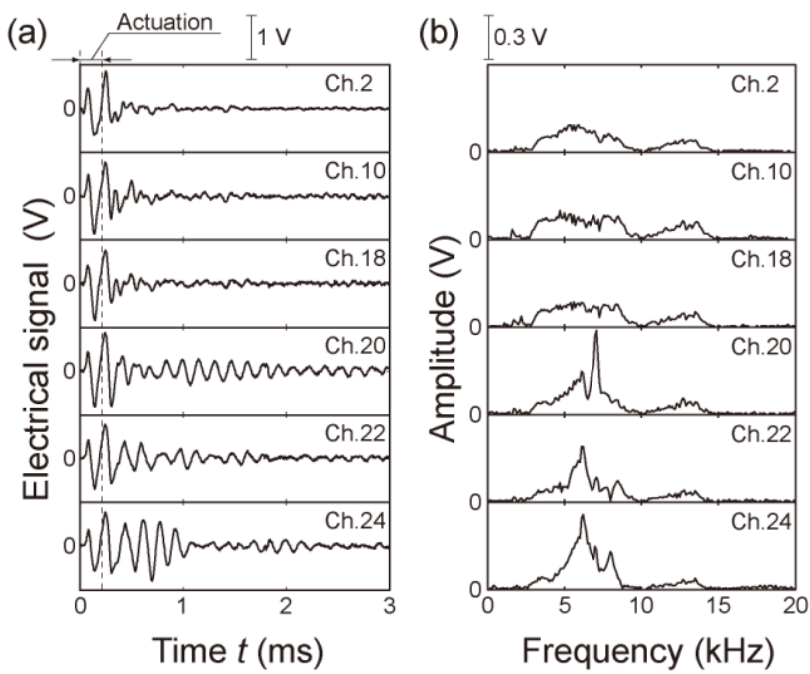

Fig. 4 (a) Electrical signals generated at $\mathrm{Ch} .2,10,18,20,22$, and 24 by applying pulsed sine wave at base frequency of $5 \mathrm{kHz}$. (b) Fourier spectra for each electrical signal.
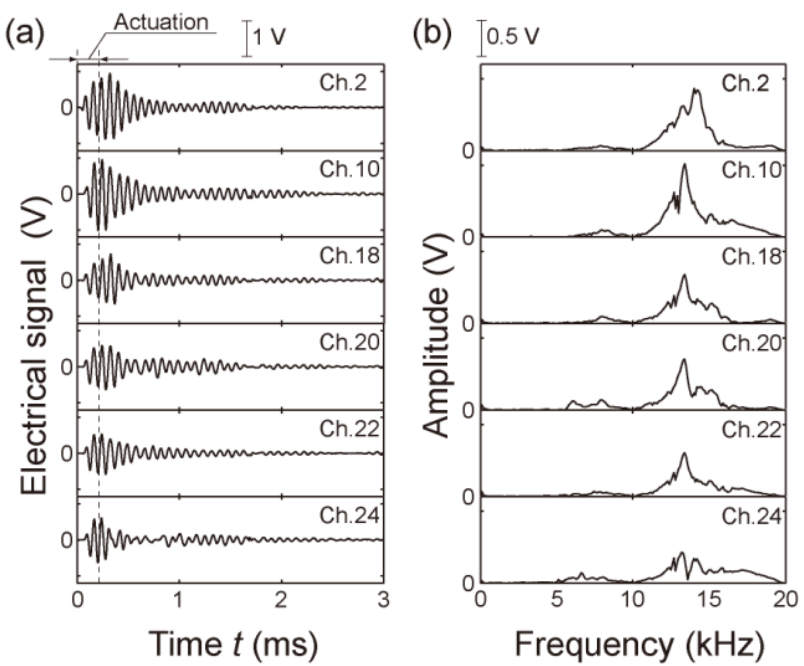

Fig. 5 (a) Electrical signals generated at $\mathrm{Ch} .2,10,18,20,22$, and 24 by applying pulsed sine wave at base frequency of $15 \mathrm{kHz}$. (b) Fourier spectra for each electrical signal. 


\subsection{Electrically Evoked Auditory Brainstem Response}

Figure 6 (a), (b), and (c) show typical eABR data obtained at various pSPLs of 96.6, 98.6, and $102 \mathrm{~dB}$, respectively. Note that the BAM generates larger electrical stimuli at higher pSPLs, as the corresponding peak-to-peak amplitudes are 2.58, 3.02, and $4.39 \mathrm{~V}$, respectively. These data are obtained using Ch. 10 at a base frequency of $10 \mathrm{kHz} . \tau$ is defined as the time elapsed from the start of electrical stimulation applied to a guinea pig. The signals during $\tau=0-0.5 \mathrm{~ms}$ correspond to the electrical stimuli from the BAM. At 96.6 $\mathrm{dB}$, observing the response in the data is difficult, as shown in Fig. 6 (a). However, at pSPLs higher than $98.6 \mathrm{~dB}$, the response is successfully and clearly observed, as shown in Figs. 6 (b) and (c) where the responses are indicated by arrows. The waves observed after $\tau$ $=1.0 \mathrm{~ms}$ and denoted by arrows in Fig. 6 (c) may correspond to the responses from different levels of the auditory nervous system such as the cochlear nucleus, superior olivary complex, lateral lemniscus, and inferior colliculi, respectively. From the above results, the developed system is shown to be capable of providing fundamental sound perception. The relationship between the pSPL and the magnitude of the response is examined in Fig. 6 (d), where the magnitude is evaluated by the amplitude $A$ of the first wave after the electrical stimulus. As shown in Fig. 6 (d), $A$ increases with pSPL, increasing rapidly from $97 \mathrm{~dB}$ and apparently saturating at the pSPLs higher than $101 \mathrm{~dB}$. There is a threshold at around 97-99 $\mathrm{dB}$ for effective stimulation. These characteristics have qualitative similarities with the relationship between the discharge rate of auditory nerve fibres and pSPL in the normal auditory system [15]. Thus, the BAM can be said to decode the pSPL into activity of the nervous system by changing the magnitude of the electrical stimuli.

To evaluate the effect of frequency selectivity of the BAM on the stimulating characteristics, eABR measurements were carried out by connecting various channels at the same pSPL of $101 \mathrm{~dB}$ and a base frequency of $10 \mathrm{kHz}$ (Fig. 7). From Fig. 7, the response magnitudes are found to change in the data with different channels. This result is due to the frequency selectivity of the BAM, where the channel near the locally resonating site (i.e., Ch. 9 and Ch.10 in this case) generates relatively large electrical stimuli compared to the other channels. Therefore, larger responses are observed with Ch. 9 and Ch.10. Thus, as a first step, the applicability of frequency selectivity is successfully demonstrated in this animal study. The value of $A$ in Fig. 6 is relatively larger than that in Fig. 7 under the same pSPL condition. We consider this difference in $A$ is due to using different guinea pigs for Fig.6 and Fig.7. Although we find the variation in the magnitude of $A$ among different guinea pigs $(n=3)$, we obtain the qualitatively similar result.

To develop a practical artificial cochlear system, the applicability must be evaluated by using a multi-channel electrode under conditions of simultaneous stimulation. This is because the frequency information is interpreted as the site of cochlear stimulation. However, the animal tests here were carried out using a single-channel electrode and the results were evaluated by the channel dependence of the response magnitude. Thus, further studies must be carried out in the future by using a multi-channel electrode to investigate whether the electrical stimulus is localized to the intended site. The problem of crosstalk between adjacent electrodes will likely be encountered in such studies. A switch circuit was introduced in this study to improve the $\mathrm{S} / \mathrm{N}$ ratio for eABR measurement. However, this circuit is unnecessary for the actual artificial cochlear system. Furthermore, the auditory nerve fibres in a healthy cochlea are known to show activities such as reverberation in response to pulsed sounds [16]. This phenomenon, which is not implemented in conventional systems, can be artificially reproduced by the BAM, as shown in Figs. 4 (a) and 5 (a). This may realize more natural hearing. The results obtained here are fundamental for the development of fully implantable artificial cochlea based on the BAM. Some remaining challenges include miniaturization of the device for implantation, amplification of electrical stimuli, optimization of electrode design[17], active feedback control for better 
frequency selectivity, mechanical tissue damage [18] during implantation, and investigations of long-term biocompatibility [19]. Among these challenges, amplification of electrical stimuli is the most essential to realize the fully implantable system. We believe integration of energy harvesting device based on microelectromechanical systems [20, 21] is one of the promising technologies to tackle this challenge. We also believe the BAM made of piezoelectric material has an advantage to develop an active feedback controlling system because it can work as a sensor and an actuator using inverse piezoelectric effect at the same time. Here, we successfully highlight the potential of the novel artificial cochlea system based on the developed BAM in animal tests.
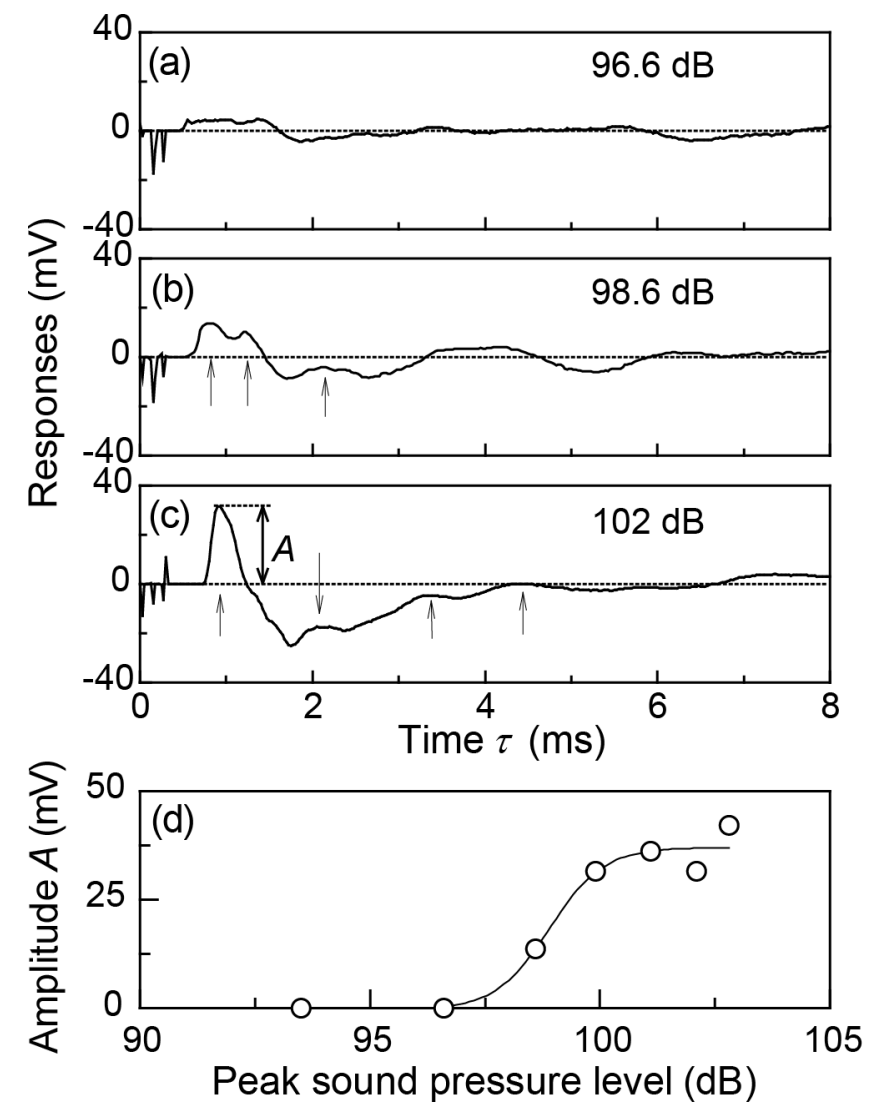

Fig. 6 eABR results by using $\mathrm{Ch} .10$ and $10 \mathrm{kHz}$ pulsed acoustic wave at peak sound pressure levels of (a) 96.6, (b) 98.6, and (c) $102 \mathrm{~dB}$, respectively. (d) Relationship between peak sound pressure levels and amplitude $A$ of first wave. 


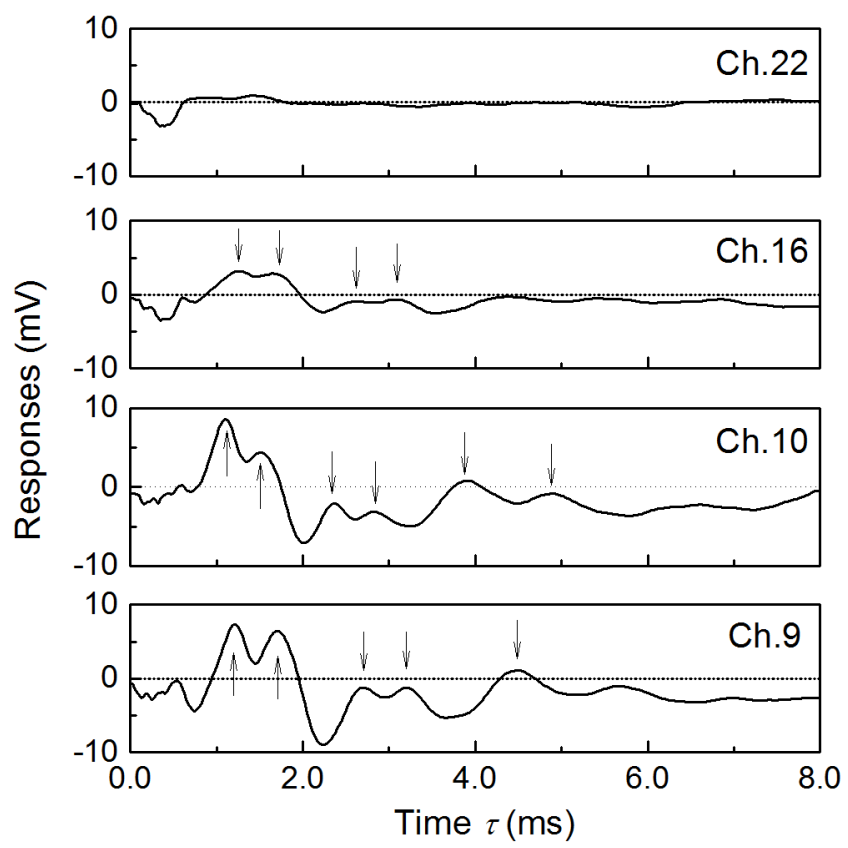

Fig. 7 Comparison of eABR results between $\mathrm{Ch} .9,10,16$, and 22 at peak sound pressure level of $101 \mathrm{~dB}$ and base frequency of $10 \mathrm{kHz}$.

\section{Concluding Remarks}

Here, we investigated the acoustic-electric conversion and frequency selectivity of the BAM by using pulsed acoustic waves to evaluate the basic applicability of the BAM to a novel artificial cochlea. Electrical signals due to the reverberation of BAM vibration were obtained from different channels and subjected to Fourier analysis to elucidate the frequency characteristics. In addition, we constructed an electrical circuit for eABR measurement that uses the signal from the BAM as the source of electrical stimulation. The measurements were conducted by electrically stimulating the auditory nerves of guinea pigs through electrodes implanted in the cochlea. Various conditions of pSPL and frequencies were used to elucidate how they influence the amplitude of the eABR signal. The sigmoidal change of the eABR signal with increasing pSPL indicated recognition of the pSPL. In addition, the relatively large stimulation-evoked response from the channel on the resonating site suggested the applicability of frequency selectivity of the BAM. The results obtained here are still at an elementary stage for the development of a novel artificial cochlea. However, we believe that they demonstrate the potential of the BAM for future development.

\section{Acknowledgement}

This work was supported by a Grant-in-Aid for Research on Sensory and Communicative Disorders from the Japanese Ministry of Health, Labor, and Welfare. The authors thank Dr. Yoichi Kagaya, Mr. Yohei Nakamoto, Mr. Masahide Hayashi, Mr. Nobuyoshi Tsuchioka, and Mr. Toshiya Kanbe of Osaka University for their technical support and valuable discussions, and Dr. Hideaki Ogita of Kyoto University for technical contributions to the eABR measurements. 


\section{References}

[1] Waltzman, S. B., 2006, "Cochlear Implants: Current Status," Expert Review of Medical Devices, 3(5), pp. 647-655.

[2] Cosetti, M. K., and Waltzman, S. B., 2011, "Cochlear Implants: Current Status and Future Potential," Expert Review of Medical Devices, 8(3), pp. 389-401.

[3] Osman, O., Shintaku, H., and Kawano, S., 2012, "Development of Micro-Vibrating Flow Pumps Using Mems Technologies," Microfluidics and Nanofluidics, pp. 1-11.

[4] Cheung, K., 2007, "Implantable Microscale Neural Interfaces," Biomedical Microdevices, 9(6), pp. 923-938.

[5] Stieglitz, T., Schuetter, M., and Koch, K. P., 2005, "Implantable Biomedical Microsystems for Neural Prostheses," Engineering in Medicine and Biology Magazine, IEEE, 24(5), pp. 58-65.

[6] Chen, F., Cohen, H. I., Bifano, T. G., Castle, J., Fortin, J., Kapusta, C., Mountain, D. C., Zosuls, A., and Hubbard, A. E., 2006, "A Hydromechanical Biomimetic Cochlea: Experiments and Models," J. Acoust. Soc. Am., 119 (1), pp. 394-405.

[7] Shintaku, H., Nakagawa, T., Kitagawa, D., Tanujaya, H., Kawano, S., and Juichi, I., 2010, "Development of Piezoelectric Acoustic Sensor with Frequency Selectivity for Artificial Cochlea," Sens. Act. A: Physical, 158(2), pp. 183-192.

[8] Tanaka, K., Abe, M., and Ando, S., 1998, "A Novel Mechanical Cochlea "Fishbone" with Dual Sensor/Actuator Charateristics," IEEE ASME Trans. Mechatron., 3(2), pp. 98-105.

[9] White, R. D., and Grosh, K., 2005, "Microengineered Hydromechanical Cochlear Model," Proc. Natl. Acad. Sci. U.S.A., 102(5), pp. 1296-1301.

[10] Wittbrodt, M. J., Steele, C. R., and Puria, S., 2006, "Developing a Physical Model of the Human Cochlea Using Microfabracation Methods," Audiol. Neurootol., 11(2), pp. 104-112.

[11] Xu, T., Bachman, M., Zeng, F. G., and Li, G. P., 2004, "Polymeric Micro-Cantilever Array for Auditory Front-End Processing," Sens. Act. A: Physical, 114(2-3), pp. 176-182.

[12] Zhou, G., Bintz, L., Anderson, D. Z., and Bright, K. E., 1993, "A Life-Sized Physical Model of the Human Cochlea with Optical Holographic Readout," J. Acoust. Soc. Am., 93(3), pp. 1516-1523.

[13] Inaoka, T., Shintaku, H., Nakagawa, T., Kawano, S., Ogita, H., Sakamoto, T., Hamanishi, S., Wada, H., and Ito, J., 2011, "Piezoelectric Materials Mimic the Function of the Cochlear Sensory Epithelium," Proc. Natl. Acad. Sci. U.S.A., 108(45), pp. 18390-18395.

[14] Ogita, H., Nakagawa, T., Lee, K. Y., Inaoka, T., Okano, T., Kikkawa, Y. S., Sakamoto, T., and Ito, J., 2009, "Surgical Invasiveness of Cell Transplantation into the Guinea Pig Cochlear Modiolus," Orl-Journal for Oto-Rhino-Laryngology and Its Related Specialties, 71(1), pp. 32-39.

[15] Delgutte, B., 1980, "Representation of Speech-Like Sounds in the Discharge Patterns of Auditory-Nerve Fibers," Journal of the Acoustical Society of America, 68(3), pp. 843-857.

[16] Goblick, T. J., and Pfeiffer, R. R., 1969, "Time-Domain Measurements of Cochlear Nonlinearities Using Combination Click Stimuli," Journal of the Acoustical Society of America, 46(4p2), pp. 924-\&.

[17] Hakamada, K., Shintaku, H., Nagata, T., Fujimoto, H., Kawano, S., and Miyake, J., 2013, "Development of a Microfabricated Device for Low-Voltage Electropermeabilization of Adherent Cells," Journal of Bioscience and Bioengineering, 115(3), pp. 314-319.

[18] Shintaku, H., Okitsu, T., Kawano, S., Matsumoto, S., Suzuki, T., Kanno, I., and Kotera, H., 2008, "Effects of Fluid Dynamic Stress on Fracturing of Cell-Aggregated Tissue During Purification for Islets of Langerhans Transplantation," Journal of Physics D: Applied Physics, 41(11), pp. 115507.

[19] Shintaku, H., Tateno, T., Tsuchioka, N., Tanujaya, H., Ito, J., and Kawano, S., 2010, "Culturing Neurons on Mems Fabricated P(Vdf-Trfe) Films for Implantable Artificial Cochlea," J. Biomech. Sci. Eng., 5(3), pp. 229-235.

[20] Morimoto, K., Kanno, I., Wasa, K., and Kotera, H., 2010, "High-Efficiency Piezoelectric Energy Harvesters of C-Axis-Oriented Epitaxial Pzt Films Transferred onto 
Stainless Steel Cantilevers," Sensors and Actuators a-Physical, 163(1), pp. 428-432.

[21] Wasa, K., Matsushima, T., Adachi, H., Kanno, I., and Kotera, H., 2012, "Thin-Film Piezoelectric Materials for a Better Energy Harvesting Mems," Journal of Microelectromechanical Systems, 21(2), pp. 451-457. 